\title{
Diffusion of nitrogen in austenitic phase: Application to nitriding of stainless steels
}

\section{Diffusion de l'azote en phase austénitique: Application à la nitruration des aciers inoxydables}

\author{
Lazhar Torchane $^{1}$, Okba Belahcene ${ }^{1}$ \\ ${ }^{1}$ Université de Tébessa, Faculté des Sciences et de la Technologie, Département de Génie Mécanique, Tébessa, Algérie.
}

\begin{abstract}
The nitriding treatment of the martensitic stainless steels aims to harden and to introduce compressive stresses on the surface of steel. Hardening is resulting of the martensitic transformation of the austenitic matrix enriched into nitrogen during cooling and of the germination and the nitride growth. In order to preserve the stainless character of the nitrided layer, it is imperative to control precipitation within the zone affected by the treatment. Our task consists in showing that is possible to control the composition of the gas atmosphere containing ammonia and argon and to carry out on the surface of nitrided samples at $1050^{\circ} \mathrm{C}$ two types of configuration of layers : a single phase layer made up by martensite enriched in nitrogen $\alpha^{\prime}{ }_{\mathrm{N}}$ and or a two phase layer made up by austenite $\gamma_{\mathrm{N}}$ and martensite $\alpha^{\prime}{ }_{\mathrm{N}}$ enriched in nitrogen.
\end{abstract}

Résumé. Le traitement de nitruration des aciers inoxydables martensitiques a pour objectif de durcir et d'introduire des contraintes de compression à la surface de l'acier. Le durcissement est issu d'une part, de la transformation martensitique de la matrice austénitique enrichie en azote au cours de la trempe et d'autre part, de la germination et de la croissance de nitrures Afin de préserver le caractère inoxydable de la couche nitrurée, il est impératif de contrôler la précipitation au sein de la zone affectée par le traitement. Notre tâche consiste à montrer qu'il est possible de contrôler la composition de l'atmosphère gazeuse à base d'ammoniac et d'argon et de réaliser à la surface d'échantillons nitrurés à $1050^{\circ} \mathrm{C}$ deux types de configuration de couches : une couche monophasée constituée de martensite enrichie en azote $\alpha_{N}^{\prime}$ et ou une couche biphasée constituée d'austénite $\gamma_{N}$ et de martensite $\alpha_{\mathrm{N}}^{\prime}$ enrichies en azote.

\section{Introduction}

L'acier inoxydable, largement utilisé dans l'industrie devient de plus en plus un produit de grande consommation. Parallèlement, il s'impose comme un matériau de base pour la fabrication des équipements industriels qui doivent résister à des sollicitations mécaniques, chimiques et thermiques de plus en plus sévères comme c'est le cas, en particulier, dans le domaine de la production électronucléaire. Pour précéder et accompagner ce développement, il est nécessaire de maîtriser parfaitement le contrôle de la microstructure des produits afin d'obtenir les caractéristiques mécaniques et physiques requises par les utilisateurs [1-5]. Notre tâche consiste à réaliser un traitement de nitruration profonde à haute température pour obtenir à la surface de l'acier étudié une couche monophasée de martensite enrichie en

\footnotetext{
a Corresponding author: torchanel@yahoo.fr
}

azote $\alpha_{N}^{\prime}$ et exempte de nitrures de chrome $\mathrm{CrN}$ dans le but d'améliorer les caractéristiques mécaniques (usure, frottement et fatigue) et de préserver le caractère inoxydable $\mathrm{du}$ matériau de base. Pour atteindre cet objectif, il est nécessaire de contrôler la vitesse de diffusion de l'azote en phase austénitique. Cela nécessite une description mathématique du flux de diffusion de cet élément dissous à travers les phases formées [6]. Ce flux peut être calculé en utilisant les lois de Fick, si le coefficient de diffusion de l'azote en phase austénitique et les concentrations limitant le domaine de stabilité de cette phase sont connus. Dans le cas de la nitruration à haute température de l'acier étudié ces paramètres ne sont pas disponibles dans la littérature [7-10]. Nous avons donc mis au point une méthodologie permettant de les déterminer expérimentalement avec une précision suffisante. Dans ces conditions, il était possible de 
calculer la courbe de prise de masse théorique de l'échantillon. Le pilotage de cette courbe théorique pendant traitement de nitruration permet de réaliser les deux types de configuration de couches visées [11].

\section{Matériau et procédure expérimentale}

Notre étude porte sur un acier inoxydable martensitique de type APX4 (NF : Z06CND16-04). Sa structure avant traitement de nitruration est constituée de martensite en lattes obtenue après austénitisation à la température de $1050^{\circ} \mathrm{C}$ et trempe à l'eau.

L'appareillage expérimental que nous utilisons est composé essentiellement (figure 1) :

- d'une thermobalance équipée d'un réacteur cylindrique vertical en alumine pourvu d'un dispositif permettant la trempe à l'eau ou à l'huile de l'échantillon,

- de débitmètres massiques pour ajuster la composition de la phase gazeuse,

- d'un micro-ordinateur assurant l'acquisition des mesures de la prise de masse expérimentale.

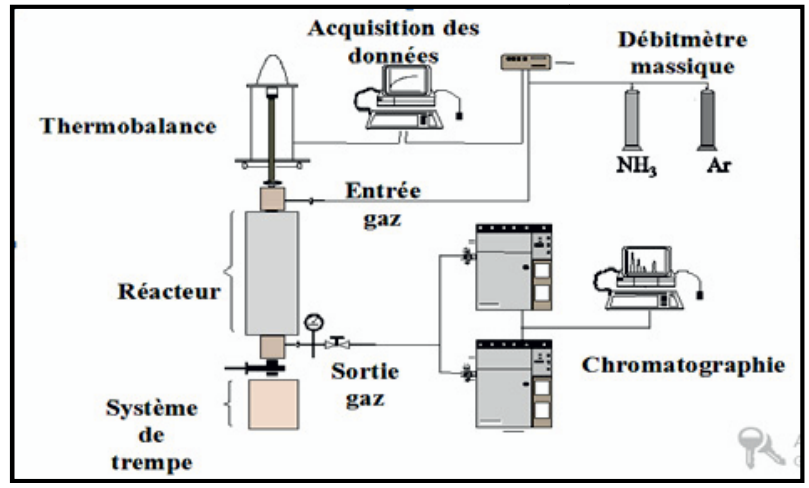

Fig.1. Représentation schématique du dispositif expérimental de nitruration.

\section{Résultats expérimentaux}

\subsection{Essai de nitruration : Configuration $\mathrm{CrN} / \gamma_{\mathrm{N}} / \alpha_{\mathrm{N}}$}

Le traitement de nitruration est réalisé à la température de $1050^{\circ} \mathrm{C}$ et à la pression atmosphérique sur un échantillon en acier Z06CND16-04.

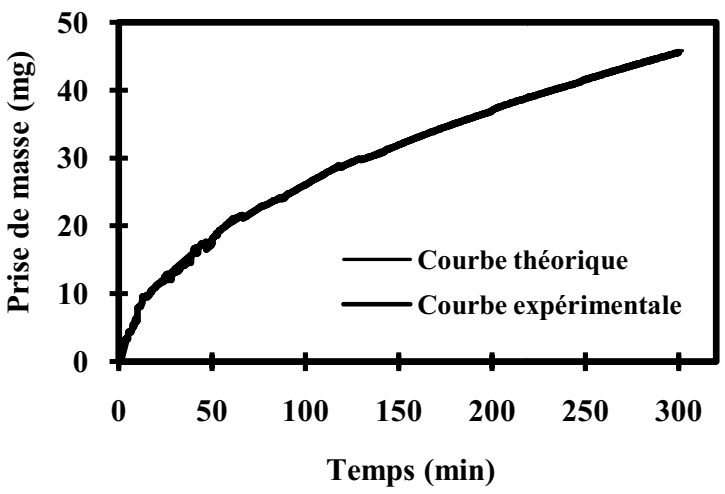

Fig.2. Variation expérimentale et théorique de la prise de masse en fonction du temps : nitruration $1050^{\circ} \mathrm{C}-5 \mathrm{~h}$ - trempe à l'eau.
Le manque de données bibliographiques relatives au coefficient de diffusion de l'azote en phase austénitique et aux concentrations limitant le domaine de stabilité de cette phase, nous a conduit dans un premier temps, à réaliser un traitement de nitruration à $1050^{\circ} \mathrm{C}$ au moyen d'un ajustement adéquat des débits de gaz assurant le suivi régulier de la courbe de prise de masse théorique calculée avec une concentration en azote de 0,5 ( $\%$ en poids) et un coefficient de diffusion de l'azote correspondant à celui du système binaire Fer- azote $\left(D_{N}=\right.$ $\left.1,8 \cdot 10^{-7} \mathrm{~cm}^{2} \cdot \mathrm{sec}^{-1}\right)$. Nous allons montrer par la suite que le couplage modèle - expérience nous permettra d'accéder au calcul précis du coefficient de diffusion de l'azote de l'acier étudié Z06CND16-04.

\subsection{Observation métallographique}

L'échantillon nitruré est découpé dans sa partie médiane, enrobé, poli jusqu'à $1 \mu \mathrm{m}$ puis attaqué avec le réactif de Catella. La micrographie optique de la figure 3 , permet d'observer plus en détail la zone la plus externe de la couche de diffusion. La présence de ferrite et /ou de martensite $\boldsymbol{\alpha}_{\mathbf{N}}^{\prime}$ en extrême surface de l'échantillon est due à une déchromisation consécutive à la précipitation dense de nitrure de chrome $\mathrm{CrN}$ dans les joints des grains de l'austénite $\gamma_{\mathbf{N}}$ et au sein même de la matrice austénitique.

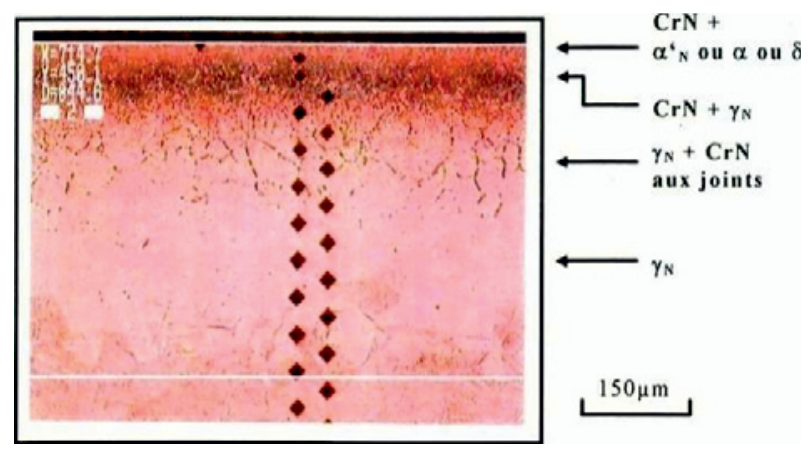

Fig.3. Coupe métallographique à plus fort grossissement de l'échantillon en acier Z06CND16 04 nitruré $5 \mathrm{~h}$ à $1050^{\circ} \mathrm{C}$.

Le tableau 1 ci-après, indique les différentes phases obtenues après traitement de nitruration à la température de $1050^{\circ} \mathrm{C}$ ainsi que leurs épaisseurs respectives.

Tableau 1 Mesures de l'épaisseur des couches de nitrures après 5 heures de nitruration à $1050^{\circ} \mathrm{C}$ et trempe à l'eau.

\begin{tabular}{|l|c|}
\hline \multicolumn{1}{|c|}{ Phases formées } & $\begin{array}{c}\text { Epaisseur des } \\
\text { couches }(\boldsymbol{\mu} \mathbf{~ m})\end{array}$ \\
\hline Nitrures $\mathbf{C r N}($ dense $)+\gamma_{\mathbf{N}}$ & $\mathbf{1 0 0}$ \\
\hline $\begin{array}{l}\text { Austénite } \gamma_{\mathbf{N}}+\mathbf{C r N} \text { aux } \\
\text { joints de grains de } \gamma_{\mathbf{N}}\end{array}$ & $\mathbf{1 0 0}$ \\
\hline Austénite $\gamma_{\mathbf{N}}$ & $\mathbf{2 5 0}$ \\
\hline Martensite à l'azote $\boldsymbol{\alpha}_{\mathbf{N}}^{\prime}$ & $\mathbf{2 0 0}$ \\
\hline
\end{tabular}

On constate en particulier, que le traitement a affecté l'échantillon sur une profondeur totale de $650 \mu \mathrm{m}$ : 
- Une couche externe biphasée constituée de nitrures de chrome $\mathrm{CrN}$ et d'austénite $\gamma_{\mathrm{N}}$ d'épaisseur voisine de $200 \mu \mathrm{m}$. La micrographie optique de la figure 3 révèle une précipitation très dense de nitrures $\mathrm{CrN}$ sur les 100 premiers micromètres de la surface. Dans l'intervalle

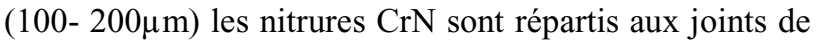
grains de l'austénite $\gamma_{\mathrm{N}}$.

- Une sous-couche monophasée de $250 \mu \mathrm{m}$ d'épaisseur, constituée de grains équiaxes qui sont caractéristiques d'une structure austénitique $\gamma_{\mathrm{N}}$ qui se différencie sans problème de la structure martensitique $\alpha_{N}^{\prime}$.

- Une couche de diffusion d'azote, de structure martensitique $\alpha_{N}^{\prime}$ et d'épaisseur égale à $200 \mu \mathrm{m}$.

\subsection{Examen radiocristallographique}

Le diffractogramme des rayons $\mathrm{X}$ de la figure 4,

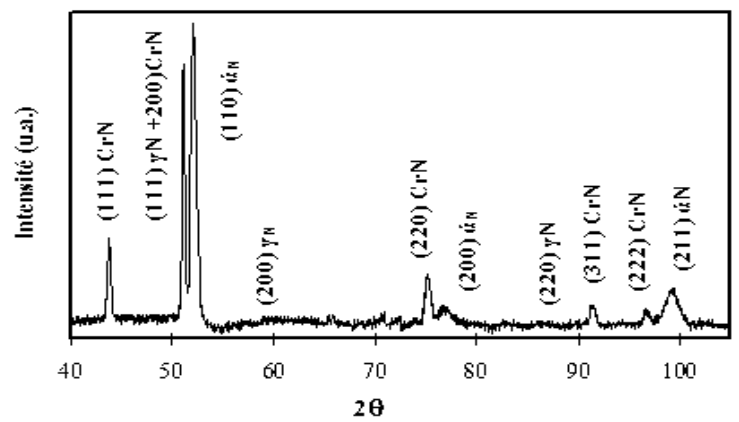

obtenue sous un angle d'incidence $\theta$ de $10^{\circ}$, confirme les observations obtenues par microscopie optique. Les pics de diffraction indiquent la présence de trois phases : nitrure de chrome CrN, austénite $\gamma_{\mathrm{N}}$ et ferrite $\alpha$-et ou $\delta$.

Fig.4. Diagramme de diffraction des rayons $\mathrm{X}$ de l'acier Z06CND16-04 nitruré 5 heures à $1050^{\circ} \mathrm{C}$ et trempé à l'eau.

\subsection{Analyse microsonde}

L'analyse par microsonde électronique a permis de déterminer avec précision la concentration en azote aux interfaces $\mathrm{CrN} /$ austénite $\gamma_{\mathrm{N}}$ et austénite $\gamma_{\mathrm{N}} /$ martensite $\alpha^{\prime}$. La figure 5 illustre les profils de concentration en azote réalisés sur les quatre faces de l'échantillon nitruré.

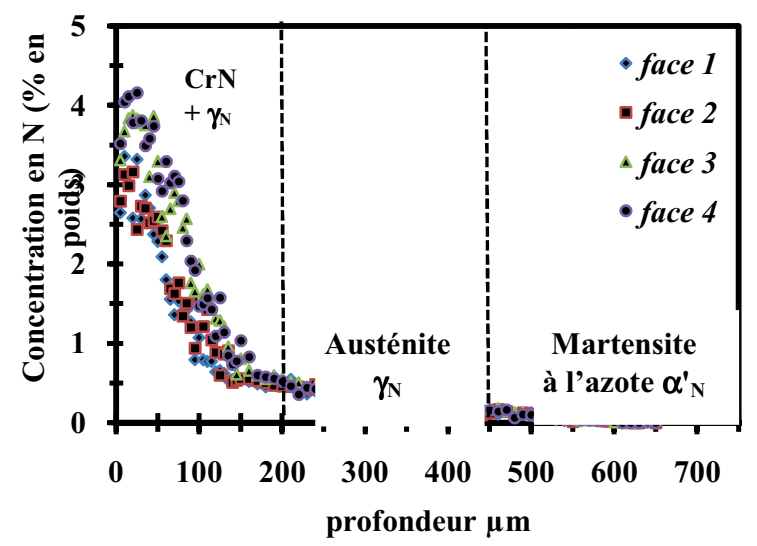

Fig.5. Profils de concentration en azote de l'acier Z06CND16-04 nitruré 5 h à $1050^{\circ} \mathrm{C}$ puis trempé à l'eau.
Les profils ainsi obtenus donnent accès aux informations suivantes :

- Le traitement de nitruration est homogène sur les quatre faces analysées et la profondeur totale de nitruration sur chacune des faces analysée est égale à $650 \mu \mathrm{m}$.

- Dans l'intervalle $(0-200 \mu \mathrm{m})$ correspondant au domaine biphasé $\mathrm{CrN}$ et $\gamma_{\mathrm{N}}$, la concentration en azote varie de 4 à 0,4 ( $\%$ en poids).

- Dans l'intervalle $(200-450 \mu \mathrm{m})$ correspondant au domaine de stabilité de l'austénite $\gamma_{\mathrm{N}}$, la concentration en azote varie de 0,4 à 0,2 ( $\%$ en poids).

- Au-delà de $450 \mu \mathrm{m}$ et sur une profondeur voisine de $200 \mu \mathrm{m}$, le gradient de concentration en azote dans la couche de diffusion $\alpha_{\mathrm{N}}^{\prime}$ varie de 0,2 à 0,01 ( $\%$ en poids).

\subsection{Estimation du coefficient de diffusion de l'azote dans l'austénite $\gamma_{\mathrm{N}}$}

A partir des profils de concentration en azote de la figure 5, nous calculons le coefficient de diffusion de l'azote en phase austénitique de l'acier Z06CND16-04 suivant la méthode de Matano [12]. Pour une profondeur $\mathbf{x}$, correspondant à la concentration $\mathbf{C}$, Matano a établi la relation suivante :

$$
D_{N}^{\gamma}(c)=-\frac{1}{2 t} \frac{\int_{0}^{c} x d c}{(d c / d x)_{c}}
$$

Avec $\mathrm{dc} / \mathrm{dx}$ la tangente à la courbe $\mathrm{C}(\mathrm{x}, \mathrm{t})$ au point $\mathrm{x}$ et $\mathrm{t}$ le temps de diffusion.

La valeur expérimentale obtenue du coefficient de diffusion est de $2.10^{-8}\left(\mathrm{~cm}^{2} \cdot \mathrm{sec}^{-1}\right)$. Cette valeur est plus faible d'un facteur 10 par rapport à celle calculée en système binaire $\mathrm{Fe}-\mathrm{N}$ à la même température de $1050^{\circ} \mathrm{C}$ : $\mathrm{D}_{\mathrm{N}}^{\gamma}=1.8 \cdot 10^{-7}\left(\mathrm{~cm}^{2} \cdot \mathrm{sec}^{-1}\right)$.

\section{Validation des résultats}

\subsection{Essai de nitruration : Configuration $\gamma_{N} / \alpha_{N}^{\prime}$}

Dans le but de valider la valeur expérimentale du coefficient de diffusion déterminée précédemment, nous réalisons un enrichissement en azote d'une durée de 5 heures avec une concentration superficielle en azote constante et égale à 0,4 ( $\% \mathrm{~N}$ en poids).

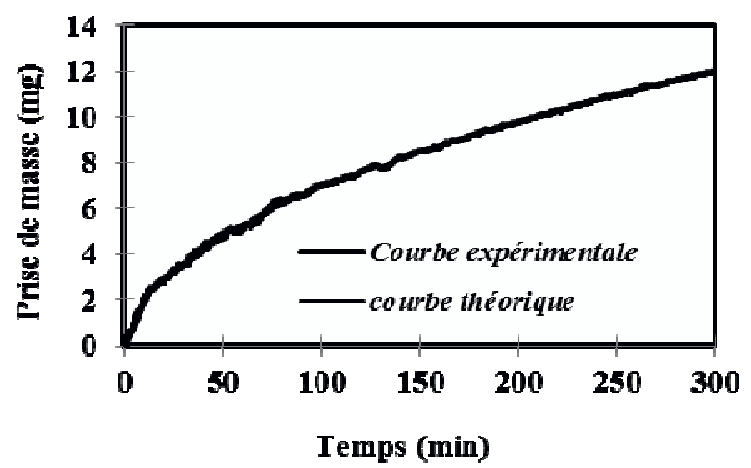

Fig.6. Courbes expérimentale et théorique de prise de masse après nitruration à $1050^{\circ} \mathrm{C}-5 \mathrm{~h}-\mathrm{C}_{\mathrm{N}}=0,4(\%$ en poids $)$. 
Cette valeur correspondant à la limite de solubilité maximale de l'azote dans l'austénite à $1050^{\circ} \mathrm{C}$ de l'acier étudié Z06CND16-04. La figure 8 présente l'évolution expérimentale et théorique de la prise de masse de l'échantillon en fonction du temps de traitement.

Le spectre de diffraction des rayons $X$ et la micrographie optique des figures 7 et 8 confirment la présence de deux phases : austénite enrichie en azote $\gamma_{\mathrm{N}}$ et martensite à l'azote $\alpha_{\mathrm{N}}^{\prime}$.

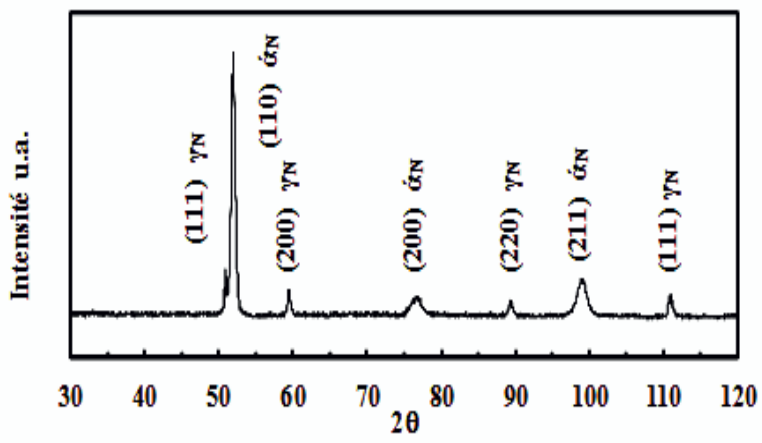

Fig.7. Diffractogramme des rayons $\mathrm{X}$ de l'acier Z06CND16-04 nitruré $5 \mathrm{~h}$ à $1050^{\circ} \mathrm{C}$ avec $\mathrm{C}_{\mathrm{N}}=0,4$ ( $\%$ en poids $)$.

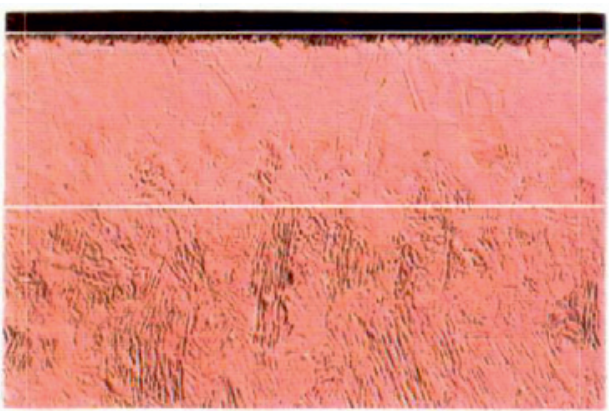

Austénite

$\gamma_{\mathrm{N}}$

Martensite

$\alpha_{\mathrm{N}}^{\prime}$

$150 \mu \mathrm{m}$

Fig.8. Micrographie optique de l'acier Z06CND16 04 après nitruration $5 \mathrm{~h}$ à $1050^{\circ} \mathrm{C}$ avec $\mathrm{C}_{\mathrm{N}}=0,4(\%$ en poids).

La figure 9 montre que la concentration superficielle moyenne en azote est égale à $0,4 \%$ en poids.

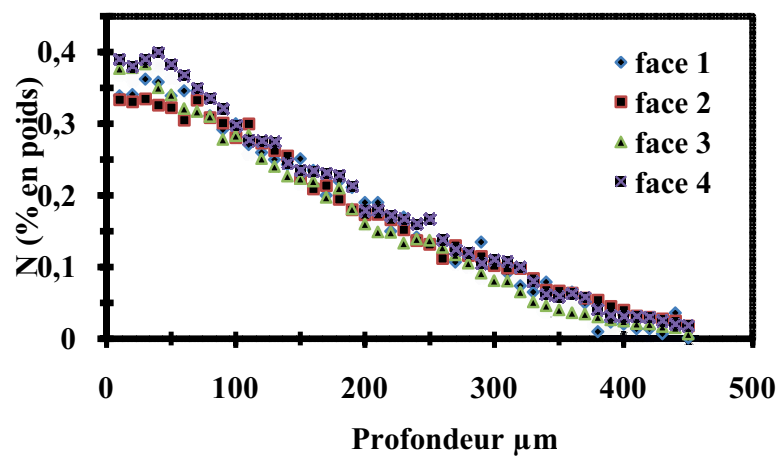

Fig.9. Profils de concentration en azote après nitruration de l'acier Z06CND16-04 $5 \mathrm{~h}$ à $1050^{\circ} \mathrm{C}$ avec $\mathrm{C}_{\mathrm{N}}=0,4$ ( $\%$ en poids).

La valeur ainsi obtenue est conforme à celle choisie pour réaliser le traitement de nitruration. Les résultats des différentes caractérisations obtenues par microscopie optique, par rayons $\mathrm{x}$ et par microsonde électronique de Castaing confirment que l'austénite est stabilisée après trempe pour une concentration en azote supérieure à 0,2 ( $\%$ en poids). Pour une teneur en azote inférieure à 0,2 ( $\%$ en poids), l'austénite se transforme en martensite $\alpha_{\mathrm{N}}^{\prime}$.

\subsection{Essai de nitruration : Configuration $\alpha_{\mathrm{N}}^{\prime}$}

Après validation expérimentale du coefficient de diffusion de l'azote et détermination précise des concentrations en azote limitant le domaine de stabilité de l'austénite à la température de $\mathbf{1 0 5 0}^{\circ} \mathbf{C}$, notre tâche consiste à présent à réaliser une couche de diffusion constituée de martensite à l'azote $\boldsymbol{\alpha}_{\mathrm{N}}^{\prime}$.

On conduit le traitement en ajustant à chaque instant les débits de gaz ammoniac et Argon introduits dans le four de la thermobalance pour suivre la prise de masse théorique calculée avec une concentration superficielle en azote :

Constante dans l'intervalle ( 0 - 5,5 heures) et égale à la limite de solubilité maximale de l'azote dans l'austénite à $1050^{\circ} \mathrm{C}: \mathrm{Cs}=0,4 \%$ en poids (figure 10),

- $\quad$ Variable dans l'intervalle $(5.5-10$ heures $)$ de 0,4 à 0,2 (\% en poids) : limite de solubilité maximale de l'azote à l'interface austénite $\gamma_{\mathrm{N}} /$ martensite $\alpha_{\mathrm{N}}^{\prime}$.

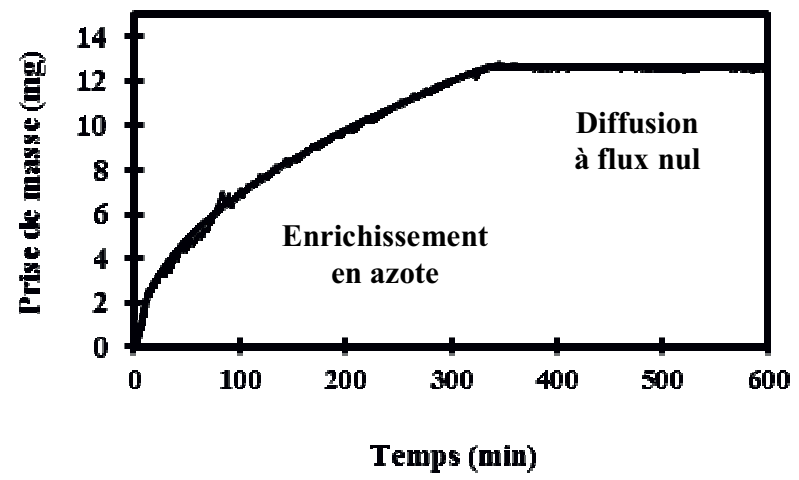

Fig.10 Courbes expérimentale et théorique de prise de masse après nitruration : $1050^{\circ} \mathrm{C}-10 \mathrm{~h}$ - concentration en azote variable de 0,4 à 0,2 ( $\%$ en poids).

Le spectre de diffraction des rayons $\mathrm{X}$ de la figure 11 et la micrographie optique de la figure 12 témoignent de la présence d'une seule phase. Ils prouvent que seule la martensite $\alpha_{\mathrm{N}}^{\prime}$ est présente dans la couche de diffusion.

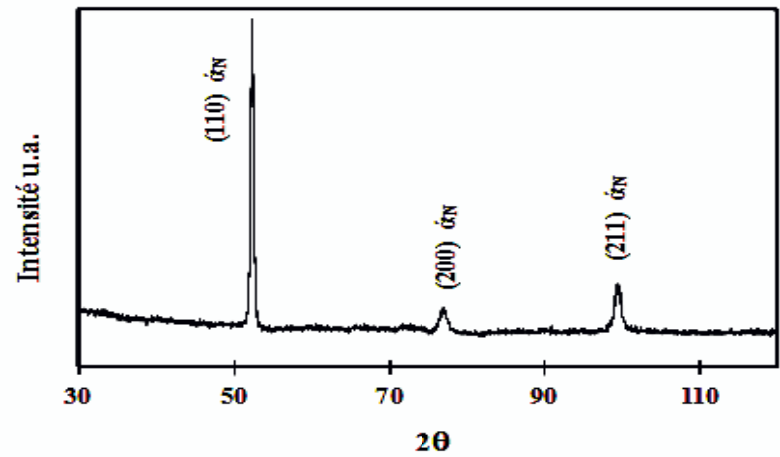

Fig.11. Diffractogramme des rayons X de l'acier Z06CND16-04 nitruré $10 \mathrm{~h}$ à $1050^{\circ} \mathrm{C}$ avec une concentration en azote variable de 0,4 à 0,2 ( $\%$ en poids). 


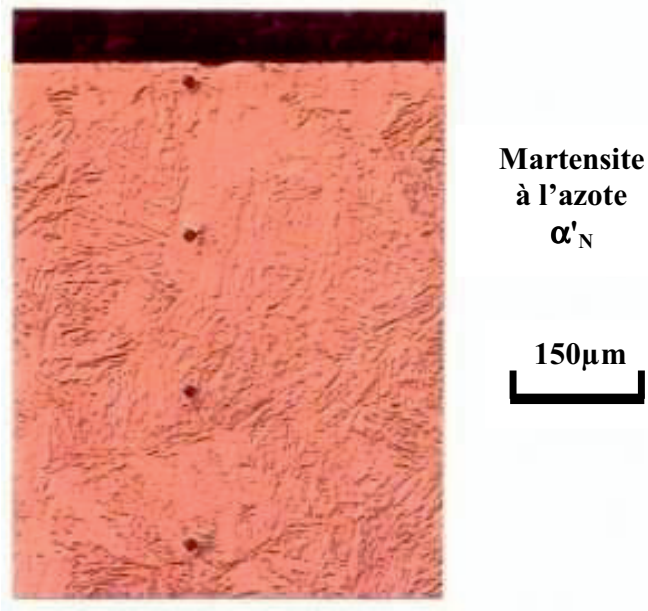

Fig.12. Micrographie optique de l'échantillon en acier Z06CND16 04 nitruré $10 \mathrm{~h}$ à $1050^{\circ} \mathrm{C}$ avec une concentration en azote variable de 0,4 à 0,2 ( $\%$ en poids).

Les profils de concentration en azote de la figure 13, réalisés sur les quatre faces de l'échantillon nitruré, font ressortir les points suivants :

- Le traitement de nitruration est homogène et la profondeur effective de nitruration obtenue sur chacune des faces analysée est voisine de $700 \mu \mathrm{m}$.

- La concentration superficielle en azote mesurée est en bon accord avec celle choisie au cours du traitement de nitruration $\mathrm{C}_{\mathrm{N}}($ surface $)=0,2(\%$ en poids $)$.

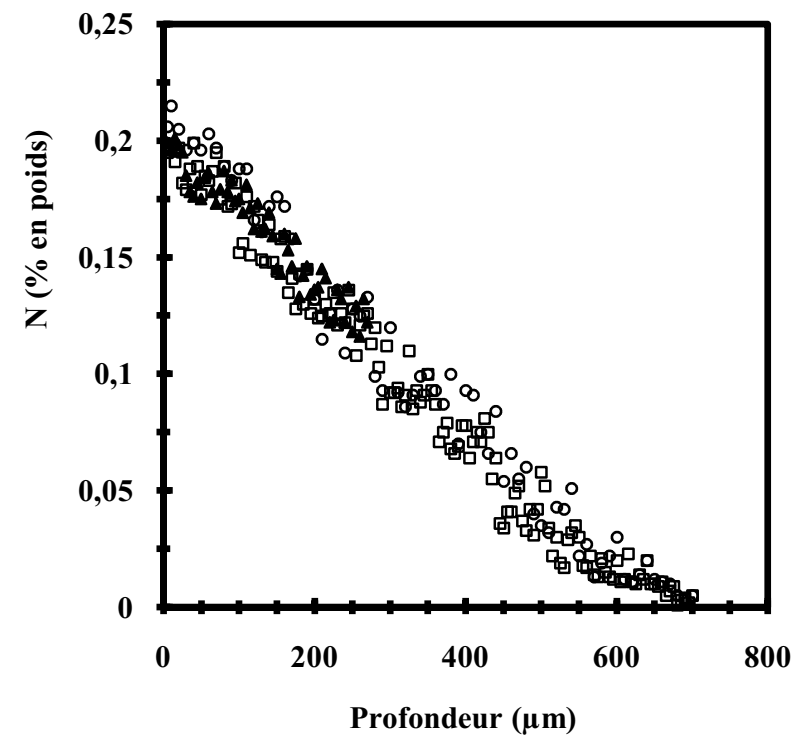

Fig.13. Profils de concentration en azote après nitruration de l'acier Z06CND16-04 $10 \mathrm{~h}$ à $1050^{\circ} \mathrm{C}$ avec une concentration en azote variable de 0,4 à 0,2 ( $\%$ en poids).

\section{Conclusion}

Dans ce travail, notre tâche a consisté de réaliser un traitement de nitruration à haute température pour obtenir à la surface de l'acier étudié Z06CND16-4 une couche monophasée de martensite enrichie en azote $\boldsymbol{\alpha}_{\mathbf{N}}^{\prime}$ exempte de nitrures de chrome de type $\mathbf{C r N}$ dans le but d'améliorer les caractéristiques mécaniques (usure, frottement et fatigue) et de préserver le caractère inoxydable du matériau de base.

Le manque de données bibliographiques relatives à notre acier, notamment le coefficient de diffusion de l'azote dans l'austénite et les concentrations en azote limitant le domaine de stabilité de cette phase à la température de $1050^{\circ} \mathrm{C}$, nous a amené dans un premier temps à mettre au point une méthodologie expérimentale à partir de laquelle nous avons déterminé expérimentalement ces paramètres.

Nous avons montré surtout que pour une teneur en azote comprise entre 0,4 et 0,2 ( $\%$ en poids), l'austénite enrichie en azote est stabilisée après trempe, on obtient la configuration austénite $\gamma_{\mathrm{N}} /$ martensite $\alpha^{\prime}$. En revanche, pour une teneur en azote inférieure ou égale à $0,2(\%$ en poids), l'austénite se transforme complètement après trempe en martensite $\alpha^{\prime}$.

La valeur expérimentale du coefficient de diffusion de l'azote en phase austénitique relatif à notre acier Z06CND16-04 est plus faible d'un facteur 10 par rapport à celle calculée en système binaire $\mathrm{Fe}-\mathrm{N}$ à la même température de $1050^{\circ} \mathrm{C}: 1,8 \cdot 10^{-7}\left(\mathrm{~cm}^{2} \cdot \mathrm{sec}^{-1}\right)$ comparée à $2.10^{-8}\left(\mathrm{~cm}^{2} \cdot \mathrm{sec}^{-1}\right)$.

\section{Références}

1. H. Berns, S. Siebert, Proc. 9th Intern. Conf. on Heat Treatment and Surface Engineering, Nizza/Frankr, 9. (1994).

2. H. Berns, S. Siebert, Randaufsticken nichtrostender StaÈhle, HTM 49, 2 (1994).

3. C. Cordier-Robert, L. Bourdeau, T. Magnin, J. Foct, Materials Letters 20, (1994).

4. H. Berns, S. Siebert, ISIJ International 36, 7 (1996)

5. H. Berns, J. Kleff, G. Krauss, R.P. Foley, Metallurgical and Material Trans. A., Vol. 27A, 7 (1996).

6. L. Torchane, Congrès International en Génie Industriel et Management des Systèmes, CIGIMS FST - FES - Maroc, 4 (2012).

7. H. Berns, J. Kleff, Stickstofflegierte nichtrostende DualphasenstaÈhle, HTM 52, 5 (1997).

8. H. Berns, V.G. Gavriljuk, Springer-Verlag, Berlin, (1999).

9. H.J. Spies, H.J. Berg, F. Vogt, B. Serchen, Mat. Wiss. Werkstofftech. (1999).

10. H. Berns, R.L. Juse, J.W. Bowman, B. Edenhofer, Heat Treatm. of Metals 2 (2000).

11. L. Torchane, Congrès International CMMS, RabatMaroc, 11 (2013).

12. Y. Adda and J. Phillibert, Tome I, Sarclay, France, (1966). 